\title{
The Prognostic Significance of Sarcopenia and the Neutrophil-to-Lymphocyte Ratio in Elderly Patients with Esophageal Squamous Cell Carcinoma
}

\section{Huajian Peng \\ Xiang Tan}

Department of Thoracic Surgery, The First Affiliated Hospital of Guangxi Medical University, Nanning, Guangxi Zhuang Autonomous Region, People's Republic of China
Correspondence: Xiang Tan Email tanxiang588@I26.com
This article was published in the following Dove Press journal:

Cancer Management and Research

Objective: To evaluate the correlation between systemic inflammation markers and sarcopenia in elderly patients with esophageal squamous cell carcinoma (ESCC) and their prognostic value.

Materials and Methods: The clinical data of 121 elderly patients with ESCC were collected. The skeletal muscle area at the level of the third lumbar vertebrae (L3) was measured by computed tomography (CT), and then the skeletal muscle index (SMI) was calculated. The neutrophil-to-lymphocyte ratio (NLR), prognostic nutritional index (PNI) and Geriatric Nutritional Risk Index (GNRI) were calculated according to laboratory standards. Univariate and multivariate Cox proportional hazards models were used to determine prognostic factors for overall survival (OS).

Results: A total of 121 elderly ESCC patients were enrolled. Among them, 65 patients had sarcopenia. NLR, PNI and GNRI are significantly related to sarcopenia. The OS of ESCC patients with sarcopenia and/or NLR $>2.24$ was significantly worse.

Conclusion: PNI, GNRI, NLR and sarcopenia were significantly related. Sarcopenia and NLR are independent prognostic factors for elderly ESCC, and when combined have better prognostic value.

Keywords: esophageal squamous cell carcinoma, sarcopenia, neutrophil-to-lymphocyte ratio, survival, nutrition

\section{Introduction}

Esophageal cancer is a highly aggressive malignancy. Its incidence rate and mortality rate are very high, ranking ninth and sixth in the world, respectively. ${ }^{1}$ The prognosis of patients with esophageal cancer is poor, and the 5-year survival rate is only $18 \% .^{2}$ The main pathological types of esophageal carcinoma include esophageal squamous cell carcinoma (ESCC) and adenocarcinoma. At present, surgical treatment is still the main treatment for ESCC. With the gradual progress of population ageing, the number of elderly patients with ESCC is gradually increasing. The treatment of elderly patients with ESCC is an important challenge. Therefore, the prognostic evaluation of elderly patients with ESCC is very important to guide the treatment plan and follow-up strategy.

The progressive growth of tumors in ESCC patients often causes the mechanical obstruction of the esophagus as well as symptoms of progressive 
dysphagia, which leads to reduced nutritional intake in ESCC patients, and then leads to cachexia. ${ }^{3}$ Many studies have focused on the nutritional status of cancer patients. The Geriatric Nutritional Risk Index (GNRI) is a simplified screening tool based on serum albumin and body weight that can be used to assess nutritionrelated risks. ${ }^{4}$ The prognostic nutritional index (PNI) is calculated by the serum albumin concentration and the total number of peripheral blood lymphocytes. ${ }^{5}$ Sarcopenia is a manifestation of body cachexia, which is mainly manifested as a gradual decline in the strength and quality of skeletal muscle. ${ }^{6}$ Because the early symptoms of esophageal cancer are often not considered, most patients can only take in liquid or are unable to eat before going to the doctor, which also causes their nutritional status to be poor. We also found that systemic inflammation is a risk factor for sarcopenia. ${ }^{7}$ Sarcopenia has been used as a predictor of poor prognosis in many gastrointestinal malignancies. ${ }^{8}$ The reduction in skeletal muscle mass measured by computed tomography (CT) is considered to be the gold standard for the diagnosis of sarcopenia. ${ }^{9}$

Complete blood cell count is an easy and inexpensive test in clinical treatment. The systemic inflammatory response is considered to play an important role in the occurrence and development of cancer. ${ }^{10}$ The systemic inflammatory response of cancer patients shows significant changes. ${ }^{11-13}$ Studies have confirmed that lymphocyte and platelet count, platelet-to-lymphocyte ratio (PLR), monocyte-to-lymphocyte ratio (MLR) and neutrophil-tolymphocyte ratio (NLR) are related to the prognosis of many cancers. ${ }^{14-16}$

The purpose of this study was to explore the relationship between preoperative systemic inflammation, GNRI, PNI and sarcopenia in elderly ESCC patients and to evaluate their correlation with the prognosis of ESCC patients.

\section{Materials and Methods}

\section{Study Population and Design}

From September 2013 to August 2017, 121 elderly patients with ESCC were recruited from the First Affiliated Hospital of Guangxi Medical University (Nanning, China). The inclusion criteria were as follows: (a) ESCC proved by pathology; (b) McKeown or Ivor-Lewis esophagectomy; (c) no distant metastasis found in preoperative examination; (d) age $\geq 65$ years; and (e) complete clinical and follow-up data. The exclusion criteria were as follows: (a) the presence of other malignant tumors; (b) incomplete resection (R1 and R2); and (c) patients with systemic infections and blood diseases. Preoperative blood test results, including complete blood count, albumin, etc., were collected. All blood samples were sent to the same laboratory for analysis. A complete blood count and measurement of height and weight were performed one week before surgery, and then the values of neutrophils $(\mathrm{N})$, platelets $(\mathrm{P})$, monocytes $(\mathrm{M})$, lymphocytes $(\mathrm{L})$ and red blood cell distribution width (RDW), NLR as N/L, MLR as $\mathrm{M} / \mathrm{L}, \mathrm{PLR}$ as $\mathrm{P} / \mathrm{L}$ were recorded. ${ }^{17}$ The PNI was calculated using the following formula: $10 \times$ serum albumin value $(\mathrm{g} / \mathrm{dl})+0.005 \times$ total lymphocyte count in the peripheral blood (per $\left.\mathrm{mm}^{3}\right){ }^{5}$ Body mass index (BMI) was calculated as the square of weight $(\mathrm{kg}) /$ height $\left(\mathrm{m}^{2}\right)$. GNRI was calculated as follows: GNRI $=1.489 \times$ serum albumin $(\mathrm{g} / \mathrm{dl})+41.7 \times$ the weight/ideal body weight. ${ }^{4}$ We use the 8th edition of the American Joint Committee on Cancer (AJCC) TNM grading system to stage patients after surgery. The study was conducted in accordance with the Declaration of Helsinki. All patients in the study provided written informed consent, and the study was approved by the Ethics Committee of the First Affiliated Hospital of Guangxi Medical College, and the Ethical approval number was 2021 (KY-E-045).

\section{Skeletal Muscle Tissue Measurement}

An enhanced CT scan of the abdomen of the patient during the week before treatment was used to collect two consecutive images at the third lumbar vertebrae (L3) level. L3 skeletal muscles include the paraspinal muscles, psoas major, rectus abdominis, internal oblique, external oblique, and transverse abdominal muscles. ${ }^{18}$ The skeletal muscle index (SMI) was calculated as the square of the skeletal muscle area $\left(\mathrm{cm}^{2}\right) /$ height $\left(\mathrm{m}^{2}\right)$. Sarcopenia was defined as the sex-specific cut-off value of $\mathrm{L} 3 \mathrm{SMI} \leq 52.4 \mathrm{~cm}^{2} / \mathrm{m}^{2}$ for males and $\leq 38.5 \mathrm{~cm}^{2} / \mathrm{m}^{2}$ for females, which correlates with mortality determined by optimal stratification. ${ }^{19}$

\section{Follow-Up}

The deadline for follow-up was December 1, 2020. The patients were followed up through outpatient follow-up visits or telephone interviews. Overall survival (OS) was defined as the time from surgery to the last follow-up or death from any cause. 


\section{Statistical Analysis}

All statistical analysis was performed using SPSS version 19.0 (SPSS Inc., Chicago, Illinois, USA), and graphs were drawn using GraphPad Prism version 5.0 (GraphPad software, Inc., La Jolla, CA, USA) and $\mathrm{R}$ version 4.0.2 (https://www.r-project.org/). The time-dependent receiver operating characteristic (ROC) curve was used to determine the clinical index cut-offs, optimal sensitivity, specificity and area under the curve (AUC). Logistic regression analysis was used to evaluate the relationship between sarcopenia and systemic inflammation indicators. KaplanMeier analysis was performed to construct survival curve. Hazard ratios (HRs) and 95\% confidence intervals (CIs) were calculated using univariate and multivariate Cox proportional hazards regression models. According to the results of univariate and multivariate Cox regression analysis, a clinical prediction model nomogram was constructed, and then the calibration curve and concordance index ( $\mathrm{C}$ index $)$ were used to evaluate the prediction accuracy of the nomogram. $\mathrm{P}<0.05$ was considered statistically significant.

\section{Results}

\section{Baseline Characteristics of the Patients and Tumors}

A total of 121 patients with ESCC were enrolled. The clinical characteristics of the patients are shown in Table 1. The median age was 69 years (65-86 years and 70.34 \pm 4.57 years). Sixty-five patients (53.7\%) had sarcopenia. We constructed the ROC curves (Figure 1A) to determine the cutoff value, sensitivity, specificity and AUC value, which were $92.9,82.8 \%, 45.6 \%$, and $0.640(95 \% \mathrm{CI}=0.541-0.740)$ for GNRI; 43.35, 85.9\%, 44.9\%, and 0.648 (95\% CI $=0.550$ 0.747 ) for PNI; and $22.49,35.9 \%, 80.7 \%$, and 0.556 (0.453-0.659) for BMI in 121 elderly ESCC patients.

\section{Systemic Inflammation Markers}

The cut-off value, sensitivity, specificity and AUC value of systemic inflammation markers were determined according to the ROC curve (Figure 1B). The cut-off value, sensitivity, specificity and AUC in our cohort were 2.24, $91.2 \%, 54.7 \%$ and $0.714(95 \% \mathrm{CI}=0.620-0.808)$ for NLR; $0.135,35.1 \%, 78.1 \%$ and $0.594(95 \% \mathrm{CI}=0.493-0.695)$ for RDW; 129.65，96.5\%， 53.1\% and $0.820 \quad(95 \%$ $\mathrm{CI}=0.747-0.892$ ) for PLR; and $0.295,94.7 \%, 40.6 \%$ and $0.635(95 \% \mathrm{CI}=0.534-0.735)$ for MLR.

\section{Correlation Between Sarcopenia and} Clinical Features

We conducted logistic regression analysis on the clinical and pathological characteristics of sarcopenia and patients and systemic inflammation indicators to confirm the relationship between them (Table 2). We found that GNRI $(\mathrm{OR}=0.436,95 \% \mathrm{CI}=0.194-0.982, \mathrm{P}=0.045), \mathrm{PNI}$ $(\mathrm{OR}=0.418,95 \% \mathrm{CI}=0.182-0.957, \mathrm{P}=0.039)$, and NLR $(\mathrm{OR}=3.750,95 \% \mathrm{CI}=1.762-7.979, \mathrm{P}=0.001)$ were significantly related to sarcopenia.

\section{Survival Analysis and Risk Factors for OS}

The follow-up period ended on December 1, 2020. A total of 57 patients died. The median follow-up period was 39.0 months (range, 1-82). Univariate Cox analysis (Table 3) showed that lymphatic metastasis, cancer embolus, degree of differentiation, tumor depth, sarcopenia, GNRI, PNI, MLR, PLR and NLR were significantly related to $\mathrm{OS}$. We performed multivariate Cox analysis on the statistically significant indicators in the univariate Cox analysis (Table 3 ). We found that sarcopenia and NLR were independent prognostic factors for elderly patients with ESCC.

Survival analysis showed that patients with sarcopenia had significantly lower OS than those without sarcopenia $(\mathrm{P}<0.0001$, Figure $2 \mathrm{~A})$, and patients with a high NLR had a significantly lower OS than patients with a low NLR $(\mathrm{P}<0.0001$, Figure $2 \mathrm{~B})$. According to the presence of sarcopenia and the NLR value $(>2.24$ or $\leq 2.24$ ), we divided the 121 elderly ESCC patients into four subgroups: sarcopenia/NLR $>2.24$, sarcopenia/ $\mathrm{NLR} \leq 2.24$, no muscle loss symptoms/NLR $>2.24$ and no sarcopenia/NLR $\leq 2.24$. We found that compared with patients in the other groups, the OS of patients with sarcopenia/NLR $>2.24$ was significantly worse $(\mathrm{P}<0.0001$, Figure 3A). We combined the two subgroups and established the sarcopenia and NLR (SNLR) group to evaluate the combined prognostic value of SNLR in elderly ESCC patients, defined as follows: no sarcopenia or $\mathrm{NLR} \leq 2.24$ was assigned 0 points $(\mathrm{N}=35)$; sarcopenia or NLR $>2.24$ was assigned 1 point $(\mathrm{N}=41)$; and both sarcopenia and NLR $>2.24$ was assigned two points $(\mathrm{N}=45)$. Survival analysis showed that patients with an SNLR score of 0 had a significantly longer OS than patients with a score of 1 or $2(\mathrm{P}<0.0001$, Figure $3 \mathrm{~B})$. 
Table I Baseline Characteristics of Patients and Tumors

\begin{tabular}{|c|c|c|c|c|}
\hline \multirow[t]{2}{*}{ Characteristics } & All Patients & Sarcopenia & Nonsarcopenia & \multirow[t]{2}{*}{$\mathbf{P}$} \\
\hline & $N=|2|$ & $N=65$ & $N=56$ & \\
\hline Age & & & & 0.959 \\
\hline$\leq 70$ & 71 & 38 & 33 & \\
\hline$>70$ & 50 & 27 & 23 & \\
\hline Gender & & & & 0.847 \\
\hline Male & 96 & 52 & 44 & \\
\hline Female & 25 & 13 & 12 & \\
\hline BMI & & & & 0.084 \\
\hline$\leq 22.49$ & 87 & 51 & 36 & \\
\hline$>22.49$ & 34 & 14 & 20 & \\
\hline Cancer embolus & & & & 0.088 \\
\hline Yes & 18 & 13 & 5 & \\
\hline No & 103 & 52 & 51 & \\
\hline Perineural invasion & & & & 0.785 \\
\hline Yes & 12 & 6 & 6 & \\
\hline No & 109 & 59 & 50 & \\
\hline Smoke & & & & 0.326 \\
\hline Yes & 42 & 20 & 22 & \\
\hline No & 79 & 45 & 34 & \\
\hline Alcohol drinking & & & & 0.905 \\
\hline Yes & 49 & 26 & 23 & \\
\hline No & 72 & 39 & 33 & \\
\hline Lymphatic metastasis & & & & 0.161 \\
\hline Yes & 58 & 35 & 23 & \\
\hline No & 63 & 30 & 33 & \\
\hline \multicolumn{5}{|l|}{ Differentiation } \\
\hline Poor & 32 & 20 & 12 & 0.092 \\
\hline Moderately & 52 & 22 & 30 & \\
\hline Well & 37 & 23 & 14 & \\
\hline Tumor depth & & & & 0.885 \\
\hline $\mathrm{TI}$ & 16 & 10 & 6 & \\
\hline $\mathrm{T} 2$ & 39 & 20 & 19 & \\
\hline T3 & 64 & 34 & 30 & \\
\hline T4 & 2 & I & 1 & \\
\hline
\end{tabular}

Note: Bold values indicate statistically significant values.

Abbreviation: BMI, body mass index.

\section{Survival Prediction Nomogram Model}

Based on the results of multivariate Cox analysis, we established a nomogram for elderly ESCC patients (Figure 4A). The results showed that NLR had the greatest impact on OS, followed by lymphatic metastasis, sarcopenia and tumor depth. At the same time, we evaluated the prognostic value of sarcopenia and NLR combined. We constructed nomograms integrating SNLR, lymphatic metastasis and tumor depth (Figure 4B). We found that SNLR was the most important factor for OS, followed by lymphatic metastasis and tumor depth. We used the Coxph method to calculate two nomograms, which had accuracy values of 0.759 and 0.761 . By establishing a 5-year OS model for the two nomograms (Figure 5A-B), we found that there was a consistency between the predicted survival probabilities and the actual survival probabilities. 

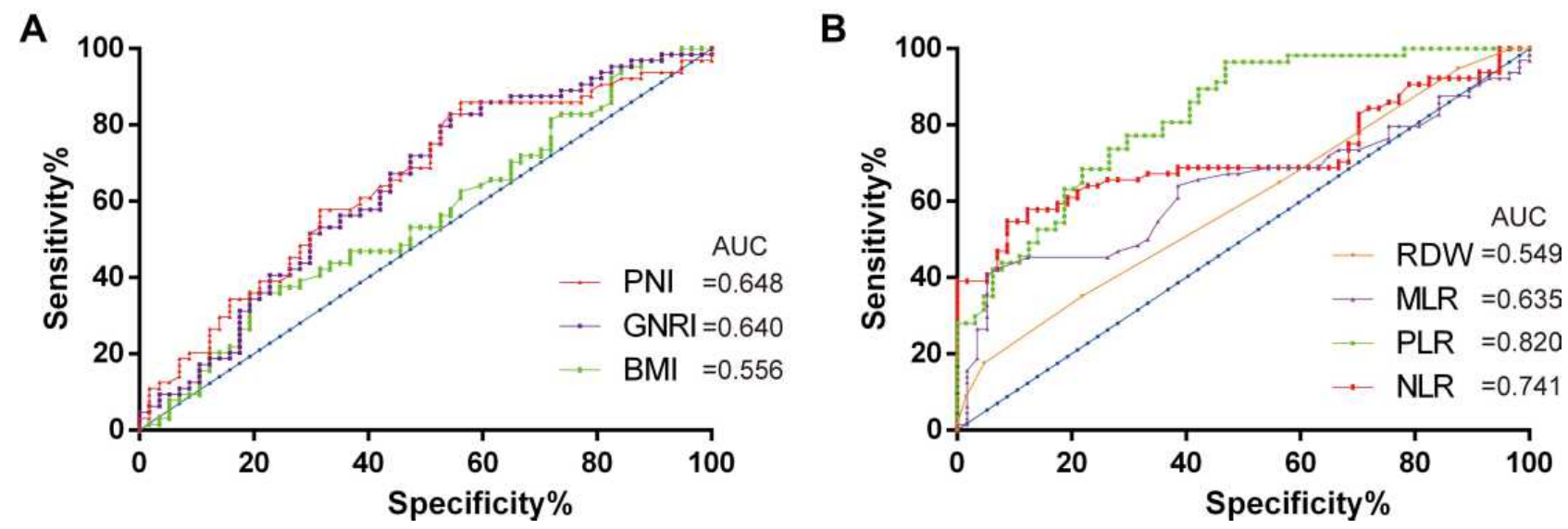

Figure I The receiver-operating characteristic (ROC) curves for (A) GNRI, BMI and PNI; (B) Inflammation index.

Abbreviations: AUC, area under the curve; BMI, body mass index, GNRI, geriatric nutritional risk index; PNI, prognostic nutritional index; MLR, monocyte-lymphocyte ratio; NLR, neutrophil-lymphocyte ratio; PLR, platelet-lymphocyte ratio, RDW, red blood cell distribution width.

\section{Discussion}

Many studies have confirmed that sarcopenia and systemic inflammation are related to the prognosis of

Table 2 Association Between Clinical Parameters and Sarcopenia

\begin{tabular}{|l|l|c|}
\hline Characteristics & OR (95\% CI) & P \\
\hline Gender(Male) & $1.091(0.452-2.634)$ & 0.847 \\
\hline Age(>70) & $1.019(0.493-2.107)$ & 0.959 \\
\hline Smoke(Yes) & $0.687(0.324-1.457)$ & 0.327 \\
\hline Alcohol drinking(Yes) & $0.957(0.462-1.980)$ & 0.905 \\
\hline BMI(>22.49) & $0.494(0.221-1.106)$ & 0.086 \\
\hline Lymphatic metastasis(Yes) & $1.674(0.813-3.446)$ & 0.162 \\
\hline Cancer embolus(Yes) & $2.550(0.848-7.67 I)$ & 0.096 \\
\hline Perineural invasion(Yes) & $0.847(0.257-2.793)$ & 0.847 \\
\hline Differentiation(Poor) & $1.630(0.712-3.728)$ & 0.247 \\
\hline Tumor depth(T3+T4) & $0.941(0.459-1.929)$ & 0.868 \\
\hline Reflux esophagitis & $1.419(0.692-2.911)$ & 0.339 \\
\hline GNRI(>92.9) & $0.436(0.194-0.982)$ & $\mathbf{0 . 0 4 5}$ \\
\hline PNI(>45.35) & $0.418(0.182-0.957)$ & $\mathbf{0 . 0 3 9}$ \\
\hline MLR(>0.295) & $1.341(0.618-2.911)$ & 0.458 \\
\hline RDW(>0.135) & $2.240(0.974-5.151)$ & 0.058 \\
\hline PLR(>I29.65) & $1.950(0.927-4.103)$ & 0.078 \\
\hline NLR(>2.24) & $3.750(1.762-7.979)$ & $\mathbf{0 . 0 0 I}$ \\
\hline No $)$ & & \\
\hline
\end{tabular}

Note: Bold values indicate statistically significant values.

Abbreviations: BMI, body mass index; MLR, monocyte-lymphocyte ratio; PLR, plateletlymphocyte ratio; NLR, neutrophil-lymphocyte ratio; GNRI, Geriatric Nutritional Risk Index; PNI, Prognostic nutritional index; RDW, red blood cell distribution width. various cancers. At present, with the ageing of the world's population, the proportion of elderly people is gradually increasing. The ageing process is related to a decline in skeletal muscle mass, and elderly individuals are more likely to suffer from sarcopenia. ${ }^{20}$ Some studies have shown that sarcopenia is not an independent prognostic factor after neoadjuvant treatment for esophageal cancer. ${ }^{21}$ However, other studies have confirmed that for elderly patients with ESCC, sarcopenia is an independent prognostic factor after neoadjuvant chemotherapy. ${ }^{22}$ Therefore, the role of sarcopenia in neoadjuvant therapy still needs further research. Some studies have confirmed the relationship between sarcopenia and the prognosis of patients with $\mathrm{ESCC}^{23-25}$ while other studies have confirmed the relationship between systemic inflammation and the prognosis of patients with ESCC. However, these studies did not explore the relationship between sarcopenia and systemic inflammation. Therefore, the relationship between sarcopenia and systemic inflammation and its prognostic role in elderly ESCC patients are still unclear. Whether there is a correlation between sarcopenia and systemic inflammation and its prognostic value are unclear. Therefore, it is necessary to study the prognostic value of sarcopenia and systemic inflammation in elderly patients with ESCC. Through research, we can confirm that NLR and sarcopenia are independent prognostic factors for elderly ESCC patients, sarcopenia is significantly related to NLR, and a higher NLR may cause sarcopenia. Through the joint survival analysis of sarcopenia and NLR, we found that ESCC patients with sarcopenia and high NLR had poor OS. By combining 
Table 3 Univariate and Multivariate Analyses of Overall Survival (OS) in the Eligible Patients

\begin{tabular}{|c|c|c|c|c|}
\hline \multirow[t]{2}{*}{ Characteristics } & \multicolumn{2}{|c|}{ Univariate Analysis } & \multicolumn{2}{|c|}{ Multivariate Analysis } \\
\hline & HR(95\% Cl) & $\mathbf{P}$ & HR(95\% Cl) & $\mathbf{P}$ \\
\hline Gender(Male) & $0.783(0.428-1.432)$ & 0.427 & & \\
\hline Age $(>70)$ & I.302(0.77I-2.197) & 0.323 & & \\
\hline Smoke(Yes) & $0.92 I(0.53|-| .598)$ & 0.769 & & \\
\hline Alcohol drinking(Yes) & $0.765(0.447-I .3 I I)$ & 0.330 & & \\
\hline $\mathrm{BMI}(>22.49)$ & $0.519(0.269-1.003)$ & 0.051 & & \\
\hline Lymphatic metastasis(Yes) & $3.482(1.969-6.159)$ & $<0.001$ & $2.050(I . I I 2-3.778)$ & 0.021 \\
\hline Cancer embolus(Yes) & $2.600(1.393-4.852)$ & 0.003 & I.164(0.565_2.397) & 0.681 \\
\hline Perineural invasion(Yes) & $0.929(0.37 I-2.327)$ & 0.875 & & \\
\hline Differentiation(Poor) & $1.879(1.082-3.262)$ & 0.025 & $1.647(0.897-3.086)$ & 0.119 \\
\hline Tumor depth(T3+T4) & $2.053(1.182-3.566)$ & 0.011 & $|.45|(0.806-2.6 \mid 4)$ & 0.215 \\
\hline Sarcopenia(Yes) & $3.885(2.088-7.226)$ & $<0.001$ & $2.344(I .21 I-4.536)$ & 0.011 \\
\hline GNRI(>92.9) & $0.399(0.236-0.674)$ & 0.001 & $0.638(0.284-1.432)$ & 0.276 \\
\hline $\mathrm{PNI}(>45.35)$ & $0.369(0.218-0.623)$ & $<0.001$ & $0.911(0.4 \mid 8-1.986)$ & 0.814 \\
\hline $\operatorname{MLR}(>0.295)$ & $2.198(1.138-4.249)$ & 0.019 & I.I70(0.546-2.507) & 0.686 \\
\hline $\operatorname{RDW}(>0.135)$ & $1.686(0.978-2.905)$ & 0.060 & & \\
\hline $\operatorname{PLR}(>129.65)$ & $3.015(1.592-5.709)$ & 0.001 & $1.364(0.633-2.942)$ & 0.428 \\
\hline $\operatorname{NLR}(>2.24)$ & $5.304(2.673-10.523)$ & $<0.001$ & $2.366(1.024-5.464)$ & 0.044 \\
\hline
\end{tabular}

Note: Bold values indicate statistically significant values.

Abbreviations: BMI, body mass index; MLR, monocyte-lymphocyte ratio; PLR, platelet-lymphocyte ratio; NLR, neutrophil-lymphocyte ratio; GNRI, Geriatric Nutritional Risk Index; PNI, Prognostic nutritional index; RDW, red blood cell distribution width.

sarcopenia and NLR, we found that elderly ESCC patients with an SNLR score of 0 had a significantly better prognosis. Through the nomogram, we found that sarcopenia had a greater impact on the OS of elderly ESCC patients, and SNLR had the greatest impact on the OS of elderly ESCC patients. Surgery is still the first
A

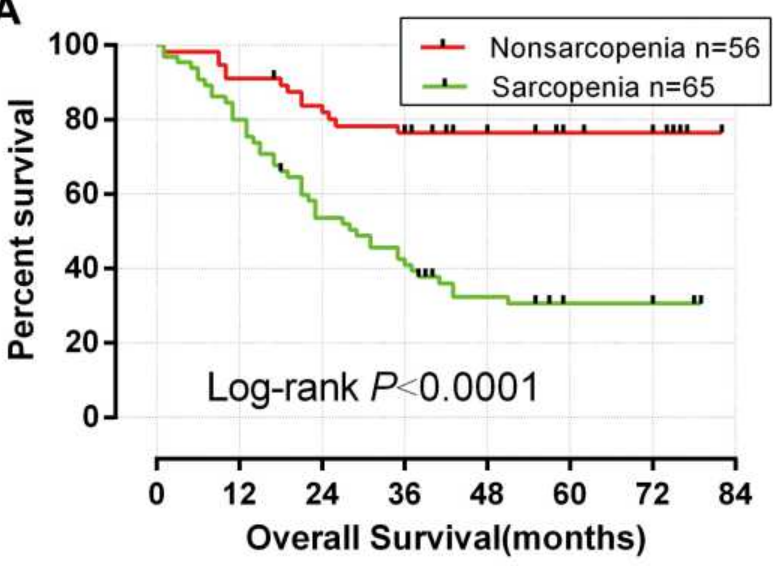

B

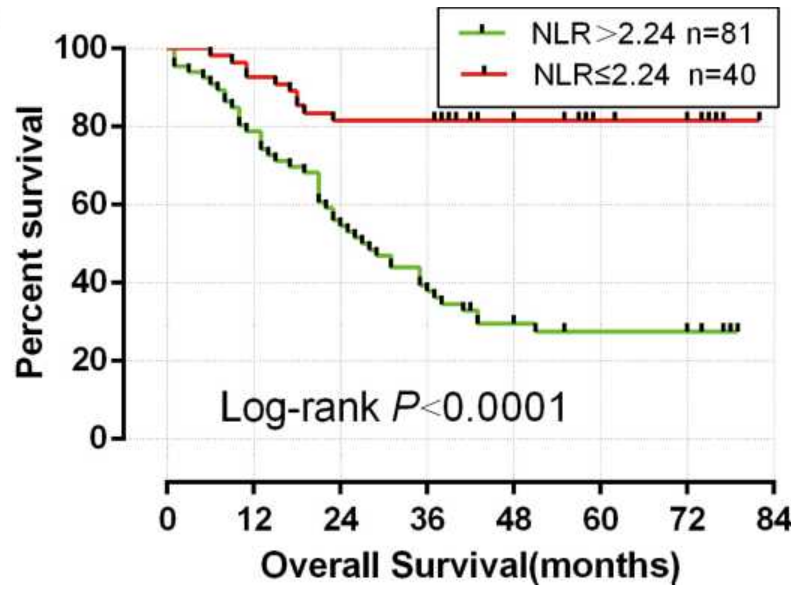

Figure 2 Kaplan-Meier survival curve of overall survival in patients: (A) Sarcopenia; (B) NLR. Abbreviation: NLR, neutrophil-lymphocyte ratio. 

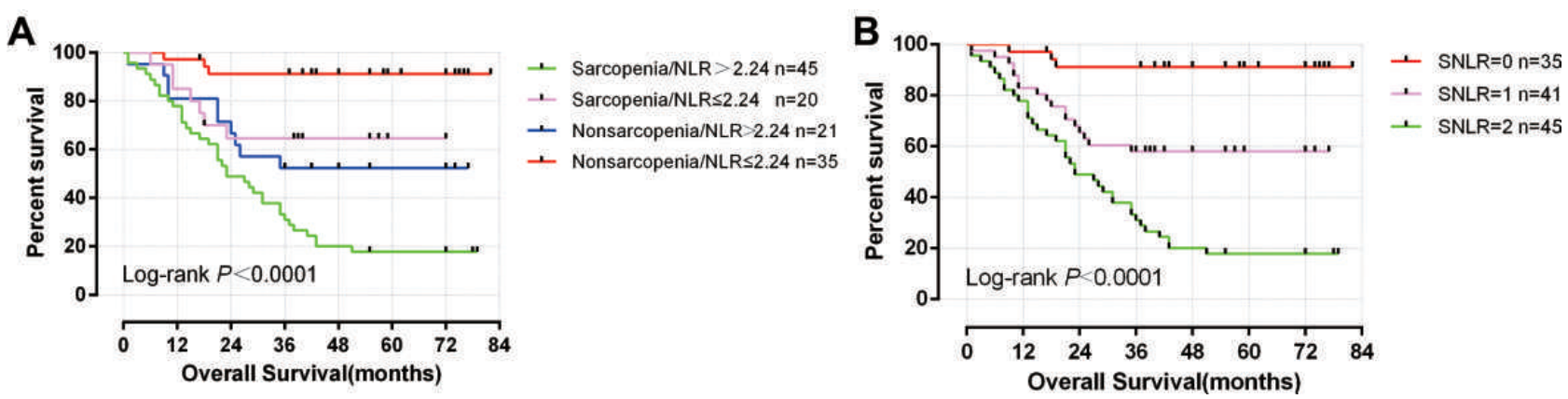

Figure 3 Kaplan-Meier survival curve of overall survival in patients: (A) Sarcopenia and NLR; (B) SNLR.

Abbreviations: NLR, neutrophil-lymphocyte ratio; SNLR, sarcopenia and the neutrophil-lymphocyte ratio.

A

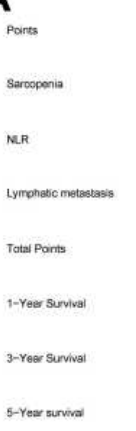

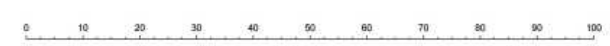
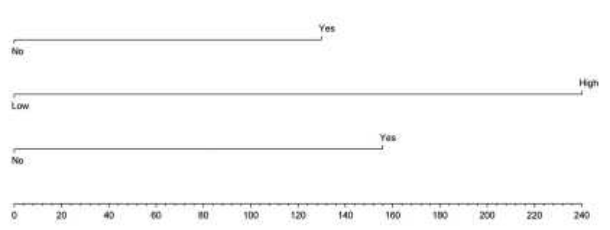

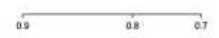

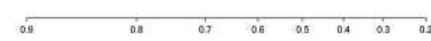

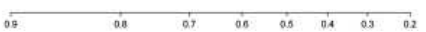

B

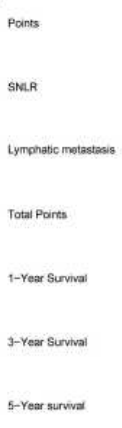

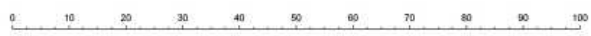
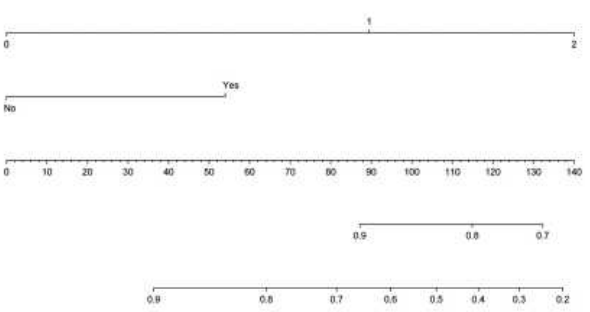

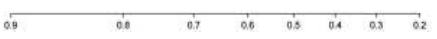

Figure 4 Nomogram for esophageal squamous cell carcinoma. The points identified on the top scale for each independent covariate were added to determine the estimated overall survival and the probability of I-, 3- and 5- year survival. (A) non-SNMR-based nomogram of overall survival. (B) SNMR-based nomogram of overall survival. Abbreviations: NLR, neutrophil-lymphocyte ratio; SNLR, sarcopenia and the neutrophil-lymphocyte ratio.
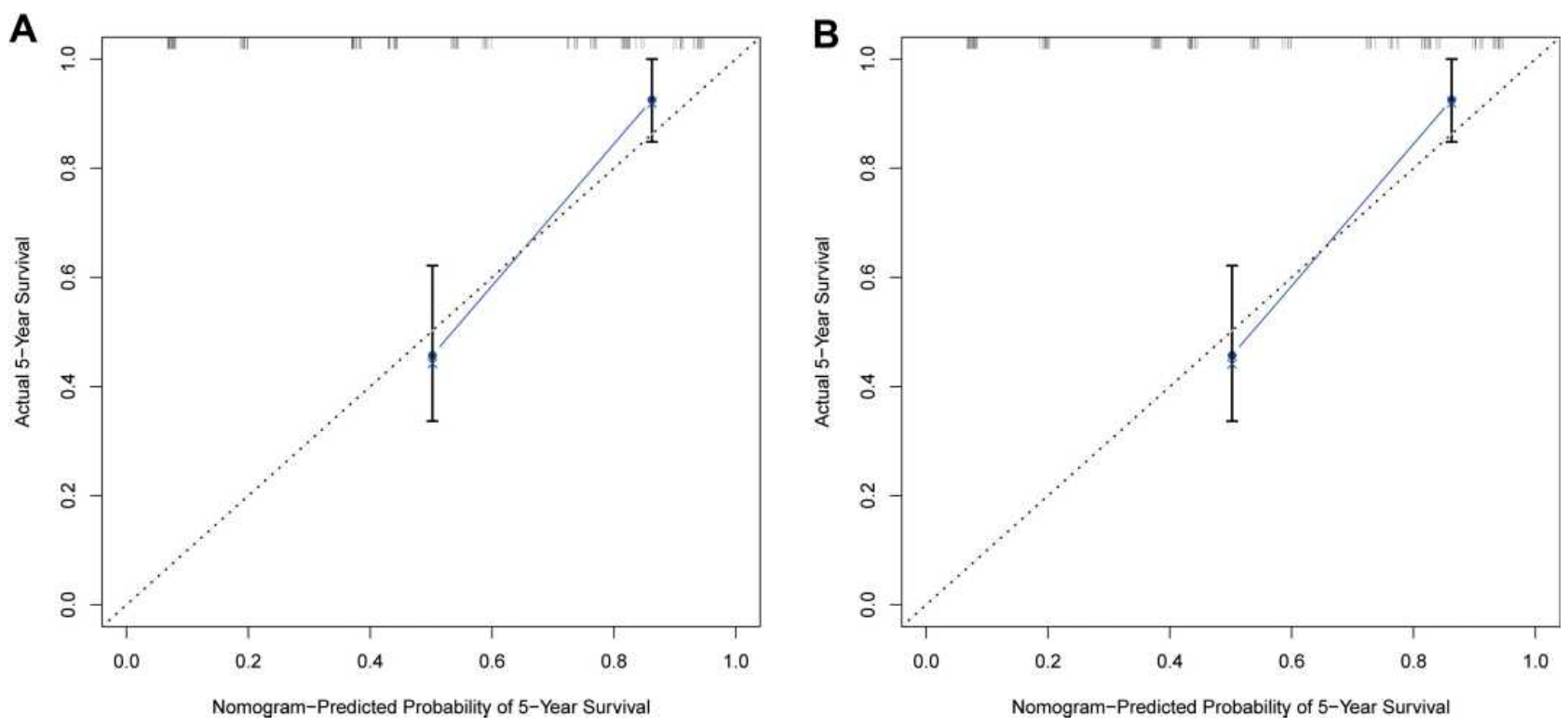

Figure 5 The calibration plot for 5-year survival of $(\mathbf{A})$ non-SNLR and (B) SNLR. The X-axis presents the predicted probability and the $Y$-axis shows the actual probability.

choice for the treatment of resectable esophageal cancer. In today's ageing population, our findings have important clinical significance.
For elderly patients with ESCC, due to the special characteristics of this population, elderly ESCC patients are prone to complications and sarcopenia during 
surgery. ${ }^{26}$ Therefore, the lack of nutrition in the elderly patients with ESCC is a problem worthy of attention. Due to the characteristics of mechanical obstruction in the esophagus caused by esophagus cancer, the incidence of malnutrition in patients with esophageal cancer is significantly higher. ${ }^{27} \mathrm{~A}$ previous study found that ESCC patients with a lower GNRI had a significantly worse prognosis, ${ }^{28,29}$ and another study found that a low PNI was significantly associated with poor prognosis in ESCC patients. ${ }^{30}$ However, in ESCC patients undergoing radical resection of esophageal cancer, the negative effects of surgery and subsequent treatment will further aggravate the original sarcopenia. Although in this study, GNRI and PNI were not independent prognostic factors for ESCC patients, sarcopenia was significantly related to PNI and GNRI. ESCC patients with low PNI and GNRI are more likely to develop sarcopenia. At the same time, sarcopenia has been indicated to have a variety of adverse effects in patients, including a decreased ability to live independently ${ }^{31}$ and an increased chance of accidental falls and fractures. ${ }^{32}$ These are all factors that lead to poor prognosis in elderly ESCC patients with sarcopenia. Therefore, in the treatment of elderly ESCC patients, a personalized and adequate treatment plan is very important.

Previous studies have found that skeletal muscle can secrete interleukin 15 (IL-15). ${ }^{33}$ IL-15 can bind to natural killer (NK) lymphocytes with high affinity through a variety of signaling pathways and protect NK cells from apoptosis by upregulating bcl-2 to ensure the normal development and survival of NK cells. ${ }^{34}$ Elderly ESCC patients with sarcopenia have a significant decrease in skeletal muscle mass, which will lead to a decrease in IL-15 secretion, resulting in a decline in the antitumor ability of the immune system, leading to tumor progression and ultimately a poor prognosis.

The relationship between sarcopenia and systemic inflammation is very close. Skeletal muscle tissue secretes numerous proinflammatory factors, such as TNF- $\alpha$ and IL6 , leading to the progression of systemic inflammation. ${ }^{34}$ However, certain proinflammatory factors can promote the decomposition of skeletal muscle and inhibit the differentiation of skeletal muscle cells by inflammatory cells and tumor cells, leading to skeletal muscle atrophy. ${ }^{35}$ This muscle atrophy caused by inflammation further aggravates systemic inflammation, leading to harmful inflammationrelated muscle weakness. ${ }^{36}$ Previous studies have also found that PNI is significantly related to tumor- infiltrating lymphocytes (TILs). TILs are a special histological feature of human cancer and reflect the individual immune response of tumors. ${ }^{30}$ Our study also found that in elderly ESCC patients, sarcopenia is significantly related to NLR, and both are independent prognostic factors for ESCC patients. Patients with a higher NLR have more significant sarcopenia. We believe that this is because systemic inflammation induces the release of proinflammatory cytokines and growth factors, which in turn has a catabolic effect on host metabolism ${ }^{37}$ and ultimately leads to a decrease in skeletal muscle mass. ${ }^{38}$

Our research has certain limitations. Our study is a retrospective study, and it was only conducted in a single center. It is necessary to conduct further largescale cohort studies to obtain more specific results and validate our findings.

\section{Conclusion}

GNRI, NRI and NLR are significantly associated with sarcopenia. Both sarcopenia and NLR are independent predictors of postoperative OS in elderly ESCC patients, and they have good comprehensive prognostic value. Sarcopenia and NLR are easily obtained clinical indicators.

\section{Acknowledgments}

This work was supported in part by the National Natural Science Foundation of China (81660387) and Development and Application of Medical and Health Appropriate Technology of Guangxi (S201654).

\section{Disclosure}

The authors declare that they have no competing interests.

\section{References}

1. Bray F, Ferlay J, Soerjomataram I, Siegel RL, Torre LA, Jemal A. Global cancer statistics 2018: GLOBOCAN estimates of incidence and mortality worldwide for 36 cancers in 185 countries. CA Cancer J Clin. 2018;68(6):394-424. doi:10.3322/caac.21492

2. Peery AF, Crockett SD, Barritt AS, et al. Burden of gastrointestinal, liver, and pancreatic diseases in the United States. Gastroenterology. 2015;149(7):1731-1741 e1733. doi:10.1053/j.gastro.2015.08.045

3. Mariette C, De Botton ML, Piessen G. Surgery in esophageal and gastric cancer patients: what is the role for nutrition support in your daily practice? Ann Surg Oncol. 2012;19(7):2128-2134. doi:10.1245/ s10434-012-2225-6

4. Bouillanne O, Morineau G, Dupont C, et al. Geriatric nutritional risk index: a new index for evaluating at-risk elderly medical patients. $\mathrm{Am}$ J Clin Nutr. 2005;82(4):777-783. doi:10.1093/ajcn/82.4.777

5. Onodera T, Goseki N, Kosaki G. [Prognostic nutritional index in gastrointestinal surgery of malnourished cancer patients]. Nihon Geka Gakkai Zasshi. 1984;85(9):1001-1005. Japanese. 
6. Cruz-Jentoft AJ, Baeyens JP, Bauer JM, et al. Sarcopenia: European consensus on definition and diagnosis: Report of the European Working Group on sarcopenia in older people. Age Ageing. 2010;39 (4):412-423. doi:10.1093/ageing/afq034

7. Wallengren O, Iresjo BM, Lundholm K, Bosaeus I. Loss of muscle mass in the end of life in patients with advanced cancer. Support Care Cancer. 2015;23(1):79-86. doi:10.1007/s00520-014-2332-y

8. Shachar SS, Williams GR, Muss HB, Nishijima TF. Prognostic value of sarcopenia in adults with solid tumours: a meta-analysis and systematic review. Eur J Cancer. 2016;57:58-67. doi:10.1016/j. ejca.2015.12.030

9. Mourtzakis M, Prado CM, Lieffers JR, Reiman T, McCargar LJ, Baracos VE. A practical and precise approach to quantification of body composition in cancer patients using computed tomography images acquired during routine care. Appl Physiol Nutr Metab. 2008;33(5):997-1006. doi:10.1139/H08-075

10. Templeton AJ, McNamara MG, Seruga B, et al. Prognostic role of neutrophil-to-lymphocyte ratio in solid tumors: a systematic review and meta-analysis. J Natl Cancer Inst. 2014;106(6):dju124. doi:10.1093/jnci/dju124

11. Balkwill F, Mantovani A. Cancer and inflammation: implications for pharmacology and therapeutics. Clin Pharmacol Ther. 2010;87 (4):401-406. doi:10.1038/clpt.2009.312

12. Zheng L, Zou K, Yang C, Chen F, Guo T, Xiong B. Inflammationbased indexes and clinicopathologic features are strong predictive values of preoperative circulating tumor cell detection in gastric cancer patients. Clin Transl Oncol. 2017;19(9):1125-1132. doi:10.1007/s12094-017-1649-7

13. Bozkaya Y, Kurt B, Gurler F. A prognostic parameter in advanced non-small cell lung cancer: the ratio of hemoglobin-to-red cell distribution width. Int J Clin Oncol. 2019;24(7):798-806. doi:10.1007/ s10147-019-01417-X

14. Wang Y, Li Y, Chen P, Xu W, Wu Y, Che G. Prognostic value of the pretreatment systemic immune-inflammation index (SII) in patients with non-small cell lung cancer: a meta-analysis. Ann Transl Med. 2019;7(18):433. doi:10.21037/atm.2019.08.116

15. Toda M, Tsukioka T, Izumi N, et al. Platelet-to-lymphocyte ratio predicts the prognosis of patients with non-small cell lung cancer treated with surgery and postoperative adjuvant chemotherapy. Thorac Cancer. 2018;9(1):112-119. doi:10.1111/1759-7714.12547

16. Watanabe K, Yasumoto A, Amano Y, et al. Mean platelet volume and lymphocyte-to-monocyte ratio are associated with shorter progression-free survival in EGFR-mutant lung adenocarcinoma treated by EGFR tyrosine kinase inhibitor. PLoS One. 2018;13(9): e0203625. doi:10.1371/journal.pone.0203625

17. Walsh SR, Cook EJ, Goulder F, Justin TA, Keeling NJ. Neutrophillymphocyte ratio as a prognostic factor in colorectal cancer. J Surg Oncol. 2005;91(3):181-184. doi:10.1002/jso.20329

18. Onishi S, Tajika M, Tanaka $\mathrm{T}$, et al. Prognostic significance of sarcopenia in patients with unresectable advanced esophageal cancer. J Clin Med. 2019;8(10):1647. doi:10.3390/jcm8101647

19. Prado CM, Lieffers JR, McCargar LJ, et al. Prevalence and clinical implications of sarcopenic obesity in patients with solid tumours of the respiratory and gastrointestinal tracts: a population-based study. Lancet Oncol. 2008;9(7):629-635. doi:10.1016/S1470-2045(08)70153-0

20. Rosenberg IH. Sarcopenia: origins and clinical relevance. J Nutr. 1997;127(5 Suppl):990S-991S. doi:10.1093/jn/127.5.990S

21. Grun J, Elfinger L, Le $\mathrm{H}$, et al. The influence of pretherapeutic and preoperative sarcopenia on short-term outcome after esophagectomy. Cancers. 2020;12(11):3409. doi:10.3390/cancers 12113409

22. Onishi S, Tajika M, Tanaka T, et al. Prognostic impact of sarcopenic obesity after neoadjuvant chemotherapy followed by surgery in elderly patients with esophageal squamous cell carcinoma. J Clin Med. 2020;9(9):2974. doi:10.3390/jcm9092974
23. Harada K, Ida S, Baba Y, et al. Prognostic and clinical impact of sarcopenia in esophageal squamous cell carcinoma. Dis Esophagus. 2016;29(6):627-633. doi:10.1111/dote.12381

24. Nishigori T, Okabe H, Tanaka E, Tsunoda S, Hisamori S, Sakai Y. Sarcopenia as a predictor of pulmonary complications after esophagectomy for thoracic esophageal cancer. J Surg Oncol. 2016;113 (6):678-684. doi:10.1002/jso.24214

25. Nakashima Y, Saeki H, Nakanishi R, et al. Assessment of sarcopenia as a predictor of poor outcomes after esophagectomy in elderly patients with esophageal cancer. Ann Surg. 2018;267(6):1100-1104. doi:10.1097/SLA.0000000000002252

26. Wagner D, DeMarco MM, Amini N, et al. Role of frailty and sarcopenia in predicting outcomes among patients undergoing gastrointestinal surgery. World J Gastrointest Surg. 2016;8(1):27-40. doi:10.4240/wjgs.v8.i1.27

27. Jordan T, Mastnak DM, Palamar N, Kozjek NR. Nutritional therapy for patients with esophageal cancer. Nutr Cancer. 2018;70(1):23-29. doi:10.1080/01635581.2017.1374417

28. Kubo N, Sakurai K, Tamura T, et al. The impact of geriatric nutritional risk index on surgical outcomes after esophagectomy in patients with esophageal cancer. Esophagus. 2019;16(2):147-154. doi:10.1007/s10388-018-0644-6

29. Migita K, Matsumoto S, Wakatsuki K, et al. The prognostic significance of the geriatric nutritional risk index in patients with esophageal squamous cell carcinoma. Nutr Cancer. 2018;70(8):1237-1245. doi:10.1080/01635581.2018.1512640

30. Okadome K, Baba Y, Yagi T, et al. Prognostic nutritional index, tumor-infiltrating lymphocytes, and prognosis in patients with esophageal cancer. Ann Surg. 2020;271(4):693-700. doi:10.1097/ SLA.0000000000002985

31. Dos Santos L, Cyrino ES, Antunes M, Santos DA, Sardinha LB. Sarcopenia and physical independence in older adults: the independent and synergic role of muscle mass and muscle function. J Cachexia Sarcopenia Muscle. 2017;8(2):245-250. doi:10.1002/ jesm. 12160

32. Schaap LA, van Schoor NM, Lips P, Visser M. Associations of sarcopenia definitions, and their components, with the incidence of recurrent falling and fractures: the longitudinal aging study amsterdam. J Gerontol a Biol Sci Med Sci. 2018;73(9):1199-1204. doi:10.1093/gerona/glx245

33. Quinn LS. Interleukin-15: a muscle-derived cytokine regulating fat-to-lean body composition. J Anim Sci. 2008;86(14 Suppl):E7583. doi: $10.2527 /$ jas.2007-0458

34. Lutz CT, Quinn LS. Sarcopenia, obesity, and natural killer cell immune senescence in aging: altered cytokine levels as a common mechanism. Aging (Albany NY). 2012;4(8):535-546. doi:10.18632/ aging. 100482

35. Lin JX, Lin JP, Xie JW, et al. Prognostic value and association of sarcopenia and systemic inflammation for patients with gastric cancer following radical gastrectomy. Oncologist. 2019;24(11):e1091e1101. doi:10.1634/theoncologist.2018-0651

36. Kalinkovich A, Livshits G. Sarcopenic obesity or obese sarcopenia: a cross talk between age-associated adipose tissue and skeletal muscle inflammation as a main mechanism of the pathogenesis. Ageing Res Rev. 2017;35:200-221.

37. Feliciano EMC, Kroenke $\mathrm{CH}$, Meyerhardt JA, et al. Association of systemic inflammation and sarcopenia with survival in nonmetastatic colorectal cancer: results from the C SCANS Study. JAMA Oncol. 2017;3(12):e172319. doi:10.1001/jamaoncol.2017.2319

38. Fearon KC, Glass DJ, Guttridge DC. Cancer cachexia: mediators, signaling, and metabolic pathways. Cell Metab. 2012;16(2):153-166. doi:10.1016/j.cmet.2012.06.011 


\section{Publish your work in this journal}

Cancer Management and Research is an international, peer-reviewed open access journal focusing on cancer research and the optimal use of preventative and integrated treatment interventions to achieve improved

The manuscript management system is completely online and includes a very quick and fair peer-review system, which is all easy to use. Visit http://www.dovepress.com/testimonials.php to read real quotes outcomes, enhanced survival and quality of life for the cancer patient. from published authors.

Submit your manuscript here: https://www.dovepress.com/cancer-management-and-research-journa 